ARTICLE

Received 28 Jun 2013 | Accepted 17 Sep 2013 | Published 16 Oct 2013 DOl: 10.1038/ncomms3629

\title{
A plasma-treated chalcogenide switch device for stackable scalable 3D nanoscale memory
}

\author{
Myoung-Jae Lee ${ }^{1}$, Dongsoo Lee ${ }^{1}$, Seong-Ho Cho ${ }^{1}$, Ji-Hyun Hur ${ }^{1}$, Sang-Moon Lee ${ }^{1,2}$, David H. Seo ${ }^{1}$, \\ Dong-Sik Kim³ ${ }^{3}$, Moon-Seung Yang ${ }^{1}$, Sunghun Lee ${ }^{1}$, Euichul Hwang ${ }^{1}$, Mohammad Rakib Uddin', Hojung Kim , \\ U-In Chung ${ }^{4}$, Youngsoo Park ${ }^{1} \&$ In-Kyeong Yoo ${ }^{5}$
}

\begin{abstract}
Stackable select devices such as the oxide $p$ - $n$ junction diode and the Schottky diode (one-way switch) have been proposed for non-volatile unipolar resistive switching devices; however, bidirectional select devices (or two-way switch) need to be developed for bipolar resistive switching devices. Here we report on a fully stackable switching device that solves several problems including current density, temperature stability, cycling endurance and cycle distribution. We demonstrate that the threshold switching device based on As-Ge-Te-Si material significantly improves cycling endurance performance by reactive nitrogen deposition and nitrogen plasma hardening. Formation of the thin $\mathrm{Si}_{3} \mathrm{~N}_{4}$ glass layer by the plasma treatment retards tellurium diffusion during cycling. Scalability of threshold switching devices is measured down to $30 \mathrm{~nm}$ scale with extremely fast switching speed of $\sim 2 \mathrm{~ns}$.
\end{abstract}

\footnotetext{
${ }^{1}$ Compound Device Lab, Samsung Advanced Institute of Technology, Samsung Electronics, Yongin, Gyeonggi-do 446-712, Korea. ${ }^{2}$ Departmant of Materials Science and Engineering, Seoul National University, Seoul 151-744, Korea. ${ }^{3}$ Department of Computer Systems \& Engineering, Inha Technical College, Incheon 402-752, Korea. ${ }^{4}$ Advanced Device Lab, Samsung Advanced Institute of Technology, Samsung Electronics, Yongin, Gyeonggi-do 446-712, Korea. ${ }^{5}$ Devices R\&D Center, Samsung Advanced Institute of Technology, Samsung Electronics, Yongin, Gyeonggi-do 446-712, Korea. Correspondence and requests for materials should be addressed to M.-J.L. (email: myoungjae.lee@samsung.com) or to S.-H.C. (email: seongho1.cho@samsung.com).
} 
T he two basic components of random access memory devices are the select switch and storage node. It is required to improve the performance of both of these elements to achieve terabit density memory. Furthermore, both components will be required to be compatible with technologies such as threedimensional (3D) cell stacking, multi-level cell, endurance and scaling for future memory and switch devices ${ }^{1,2}$. Resistive random access memory has been considered to be one of the most promising candidates to overcome scaling limits of the conventional memory due to its scalability, data retention (nonvolatility), fast switching speed and low power consumption ${ }^{3-15}$. In a high-density memory system such as dynamic random access memory, a select device is required to suppress sneak current paths ${ }^{3,8,9}$. Current select devices have been Si-based transistors to obtain sufficient on-current density and reliability. However, the three-terminal Si transistors are not suitable for 3D crossbar stacked structure due to their high processing temperature and difficulty in both scaling and stacking ${ }^{8,9}$. Recently, various bidirectional select devices, for instance the varistor-type switch $^{16}$ and mixed-ionic-electronic-conduction device ${ }^{17}$ have been proposed for bipolar resistive memory applications. The threshold switching (TS) devices are able to overcome the previous limitations by imitating bidirectional diode-like behaviour while being composed of a single compound and providing sufficient current density. Initially, oxide materials such as $\mathrm{V}_{2} \mathrm{O}_{5}$ (refs 18,19$)$ and $\mathrm{NiO}_{2}$ (ref. 20) as well as several chalcogenide materials ${ }^{21,22}$ have been investigated as TS materials. The chalcogenide material, As-Te-Ge-Si, despite its good TS characteristics, has had key issues on degradation with repeated cycling and reliability at postprocessing temperatures of $500-600^{\circ} \mathrm{C}^{21,23}$. In this report, we present two nitrogen $\left(\mathrm{N}_{2}\right)$ processes, reactive $\mathrm{N}_{2}$ sputtering and plasma hardening, which together form a thin, highly crosslinked glass $\mathrm{Si}_{3} \mathrm{~N}_{4}$-based barrier that suppresses $\mathrm{Te}$ diffusion in the chalcogenide film, leading to dramatic improvement on its endurance and switching distribution. We believe that a similar treatment is applicable to any materials in which elemental diffusion limits reliability and cycle-to-cycle distribution. Especially, the introduction of reactive $\mathrm{N}_{2}$ during deposition improves the thermal stability of the chalcogenide glass, allowing for the memory node and switch device to be integrated into a single stack in contrast with previous studies that utilized a wire-bonded connection or another external method of combining switching devices and memory nodes $8,19,24$.

\section{Results}

Resistive memory and TS devices. Due to the single material composition and ease of deposition onto arbitrary substrates, TS devices are ideal for stacked 3D memory structures, as shown in Fig. 1a. The scheme presented here stacks a memory element with a switch element between crossbar metal electrodes, which allows the highest cell geometric density. We demonstrate the $I-V$ characteristics of the As-Te-Ge-Si-N switch and the $\mathrm{TaO}_{\mathrm{y}} /$ $\mathrm{Ta}_{2} \mathrm{O}_{5-\mathrm{x}}$ memory node for $(0.5 \mathrm{um})^{2}$ cell sizes, respectively, in Fig. 1b,c. In the switch device, we observe a volatile TS with
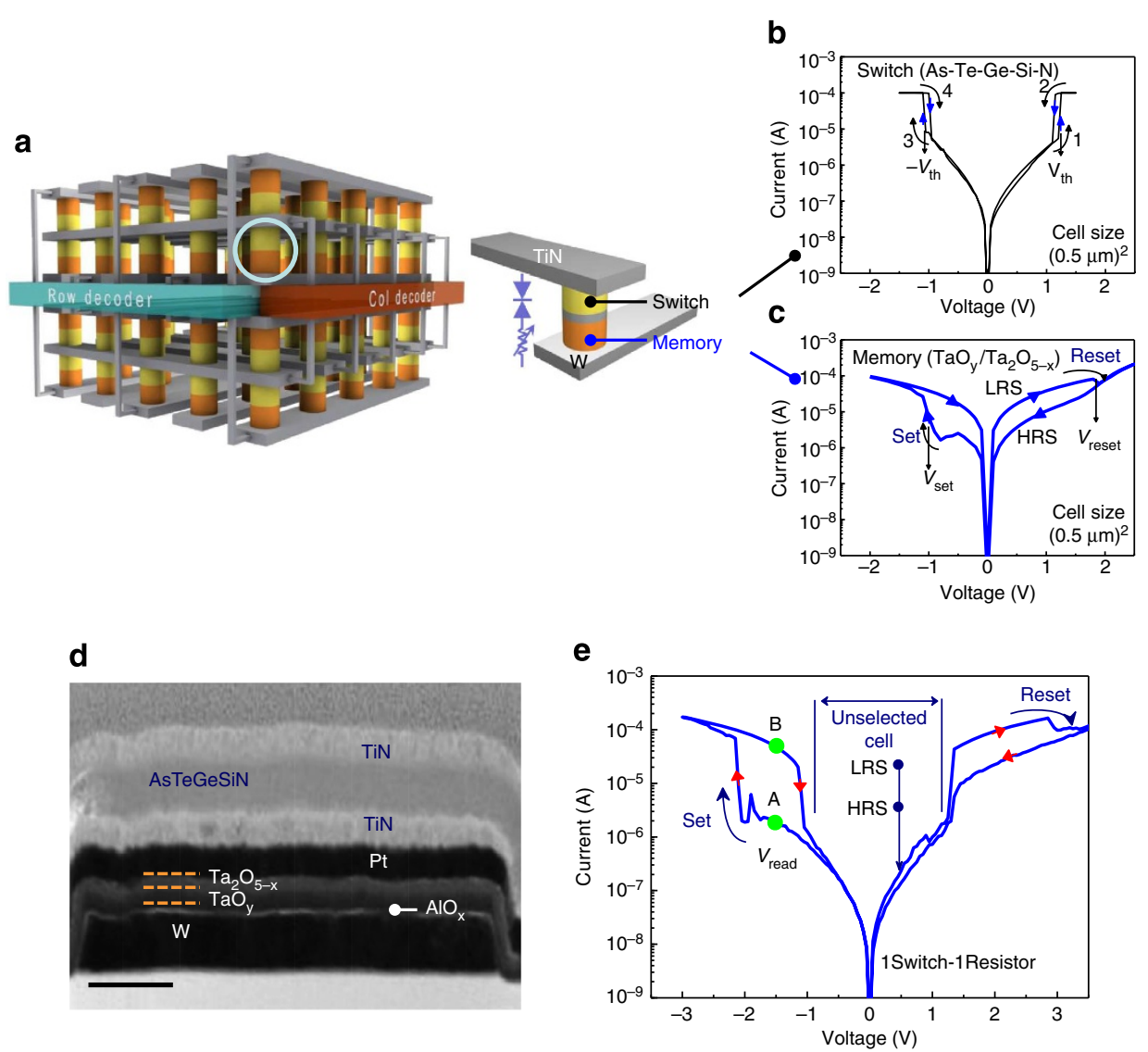

Figure 1 | $\mathrm{TaO}_{\mathbf{y}} / \mathrm{Ta}_{\mathbf{2}} \mathrm{O}_{5-\mathbf{x}}$ resistive switching and As-Te-Ge-Si-N TS devices. (a) Idealized schematic of 3D-stacked memory structure using switch and memory element in crossbar array structure with enlarged view of memory cell. (b) I-V TS operation of the switch device (As-Te-Ge-Si-N) in the crossbar array. (c) I- $V$ non-volatile switching operation of the memory device $\left(\mathrm{TaO}_{y} / \mathrm{Ta}_{2} \mathrm{O}_{5-\mathrm{x}}\right)$ in the crossbar array. (d) A cross-sectional TEM image of the 1S-1R with W/AIO $/ \mathrm{TaO}_{y} / \mathrm{Ta}_{2} \mathrm{O}_{5-x} / \mathrm{Pt} / \mathrm{TiN} / \mathrm{As}-\mathrm{Te}-\mathrm{Ge}-\mathrm{Si}-\mathrm{N} / \mathrm{TiN}$-stacked structure. Scale bar, $100 \mathrm{~nm}$. (e) I-V characteristics of combined switch and memory device structure described with reduced leakage current at below $\pm 1 \mathrm{~V}$ (readout margin for data reading in crossbar array). 
threshold voltage of $\pm 1 \mathrm{~V}$. Meanwhile, the memory device shows non-volatile bipolar resistive switching between a high resistance state (HRS) and a low-resistance state (LRS) with the $V_{\text {set }}=-1 \mathrm{~V}$ and $V_{\text {reset }}=+2 \mathrm{~V}$.

Figure 1d shows a cross-sectional transmission electron microscopy image of the stacked structure that combines our memory and select device. The $\mathrm{TaO}_{\mathrm{y}} / \mathrm{Ta}_{2} \mathrm{O}_{5-\mathrm{x}}$ bilayer memory element has been previously demonstrated to show non-volatile high endurance up to $10^{12}$ and considered to be an appropriate material for the study of switch elements ${ }^{3}$. The structure begins with the bottom $\mathrm{W}$ electrode onto which a thin $2 \mathrm{~nm}$ buffer layer is deposited by atomic layer deposition. This $\mathrm{AlO}_{\mathrm{x}}$ buffer layer is used to reduce excessive voltage drop across the switch and memory node when they are both in their respective LRS $^{3}$. In addition, the thin $\mathrm{AlO}_{\mathrm{x}}$ layer suppresses any chemical reaction between the $\mathrm{W}$ electrode and the memory node layer ${ }^{3}$. Next, the $\mathrm{TaO}_{\mathrm{y}}(20 \mathrm{~nm}) / \mathrm{Ta}_{2} \mathrm{O}_{5-\mathrm{x}}(10 \mathrm{~nm})$ bilayer memory stack is deposited as a storage element. The middle electrode is a $\mathrm{Pt} / \mathrm{TiN}$ double layer contacted to the appropriate sides for optimal switching ${ }^{3}$. The As-Te-Ge-Si-N chalcogenide TS layer is then deposited. To form a highly crosslinked glass $\mathrm{Si}_{3} \mathrm{~N}_{4}$-based barrier using the $\mathrm{Si}$ within the switching material, we subsequently use a $\mathrm{N}_{2}$ plasmahardening process on the deposited chalcogenide layer. Finally, the TiN top electrode is formed to complete the stacked device. Figure $1 \mathrm{e}$ shows the $I-V$ characteristics of the combined one switch-one resistor (1S-1R) stack structure (Supplementary Fig. S1 also shows the multiple cycling to demonstrate the combined switching concept). For combined operation of the switch and resistor, the set and reset voltages $V_{\text {set }}$ and $V_{\text {reset }}$ of the resistor should ideally both be larger than the threshold voltage of the switch device $\pm V_{\text {threshold }}$. The reading operation is performed at $V_{\text {read }}$ of $-1.5 \mathrm{~V}$ where the largest on/off ratio occurs. When the resistance device is in the off-state, the current value at $A$ will be read, whereas when the resistance is in the onstate, the current value $B$ will be read. In both previous cases, the reading voltage is chosen so the specific switch device is turned on, whereas adjacent switch devices in a theoretical array are kept off. In both reading cases, the switch is turned on so that the non- volatile memory device determines the reading current. As the memory is scaled down and better materials and processes are developed to reduce the on-state (and off-state current), the reading current will correspondingly decrease.

As-Ge-Te-Si and As-Ge-Te-Si-N switch devices. To write to the $1 \mathrm{~S}-1 \mathrm{R}$ device $V_{\text {set }}$ of $-2 \mathrm{~V}$ is applied, which turns on both the switch device and sets the resistance device to the LRS. Meanwhile, for the erase operation of the $1 \mathrm{~S}-1 \mathrm{R}$ device, a $V_{\text {reset }}$ of $+3 \mathrm{~V}$ is applied, which turns on the switch device and sets the resistance device to the HRS. To integrate further devices in a stack, we could start $\mathrm{W}$ deposition again and duplicate the structure repetitiously.

One should note that the highest temperature during stack fabrication occurs for the $\mathrm{TaO}_{\mathrm{y}} / \mathrm{Ta}_{2} \mathrm{O}_{5-\mathrm{x}}$ memory storage layer at $\sim 400^{\circ} \mathrm{C}$. For pristine As-Ge-Te-Si switches, without reactive $\mathrm{N}_{2}$, severe thermal degradation takes place as compared in Fig. 2a,b. After annealing As-Ge-Te-Si switches at $500^{\circ} \mathrm{C}$ in vacuum the device failed after just a few cycles. In addition, the optimal $\mathrm{N}_{2}$ amount was sensitive down to just $1 \mathrm{sccm}$ during $\mathrm{N}_{2}$ reactive sputtering. Figure $2 \mathrm{c}$,d confirms that with $1 \mathrm{sccm}$ for reactive $\mathrm{N}_{2}$ introduced, although the initial properties were relatively unaffected, the thermal stability was greatly improved and cycling with a good distribution was demonstrated. Further increasing the $\mathrm{N}_{2}$ amount to $2 \mathrm{sccm}$ slightly degraded the switching distribution, whereas past $10 \mathrm{sccm}$ the As-Ge-Te-Si-N film did not show reliable switching (Supplementary Fig. S2). The reactive $\mathrm{N}_{2}$ process allowed for a fully integrated $1 \mathrm{~S}-1 \mathrm{R}$ device to be investigated and properties of the stackable cell to be verified. Further elucidation is required into the role of $\mathrm{N}_{2}$ in the films, which acts to thermally stabilize the chalcogenide glass; however, it may be related to $\mathrm{Si}_{3} \mathrm{~N}_{4}$ within the film, which retards crystallization of the chalcogenide material and affects the diffusivity of $\mathrm{Te}^{23}$; (Supplementary Fig. S3).

The $I-V$ measurements of the complete $1 \mathrm{~S}-1 \mathrm{R}$ structure in stacked cell, namely, W/ $\mathrm{AlO}_{\mathrm{x}} / \mathrm{TaO}_{\mathrm{y}} / \mathrm{Ta}_{2} \mathrm{O}_{5-\mathrm{x}} / \mathrm{Pt} / \mathrm{TiN} / \mathrm{As}-\mathrm{Te}-\mathrm{Ge}-$ $\mathrm{Si}-\mathrm{N} / \mathrm{TiN}$ are shown in Fig. 1e to confirm that processing did not

c

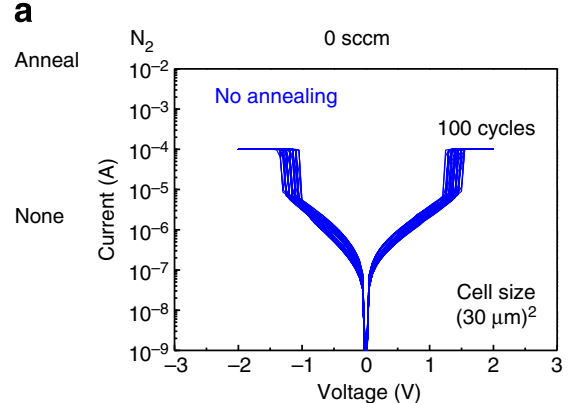

b

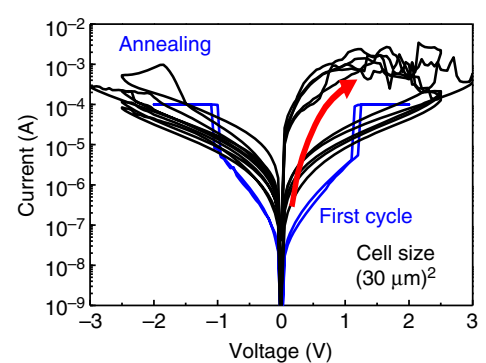

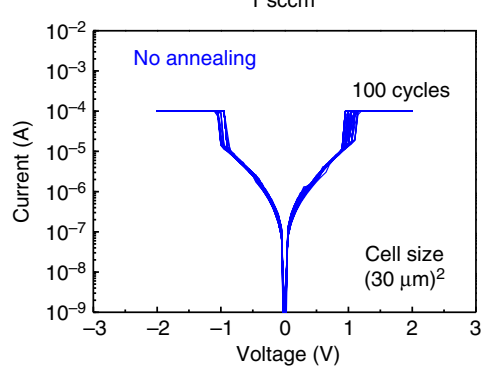

d

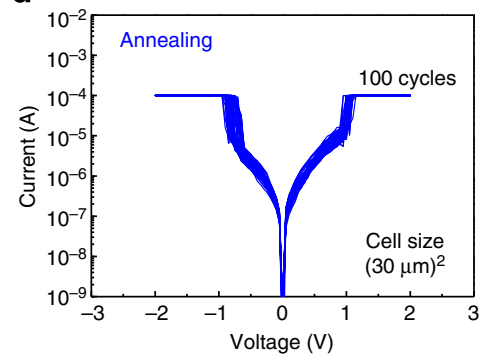

Figure 2 | Comparison of electrical cycling between both annealed and unannealed As-Ge-Te-Si and As-Ge-Te-Si-N switch devices. (a) As-deposited As-Ge-Te-Si, (b) annealed As-Ge-Te-Si, (c) as-deposited As-Ge-Te-Si-N and (d) annealed As-Ge-Te-Si-N switch devices. These data show 100 switching cycles for each device except $\mathbf{b}$. 
cause damage to the device. The change from HRS to LRS (set) occurs at $-2.2 \mathrm{~V}$, whereas the opposite process (reset) occurs at $+2.8 \mathrm{~V}$. At below $\pm 1 \mathrm{~V}$, the TS device is off so that no programming can occur. The behaviour of the switch and the memory layers are clearly demonstrated in a single integrated stack. It is important to note that the leakage current level at a voltage of below $\pm 1 \mathrm{~V}$ (off state of TS device) determines the possible array size for memory that can be achieved as stray current paths must be blocked (this is further explained by using a $1 / 3-\mathrm{V}$ programming method in the Supplementary Fig. S4). More information regarding memory array size and the stray leakage path issues are presented in the Supplementary Fig. S5. The on/off ratio of the combined memory cell was about two orders of magnitude as read in the negative biasing conditions at $-1.5 \mathrm{~V}$, as shown in Fig. 1e (green circles).

XPS analysis and scalability of As-Ge-Te-Si-N switch devices. We further investigated the properties of the select device and the effects of the previously mentioned $\mathrm{N}_{2}$ plasma-hardening process, which led to a significant improvement in cycle-to-cycle switching distribution. The depth profiles of X-ray photoelectron spectroscopy (XPS) were used to analyse the film composition. The depth profile of a pristine As-Te-Ge-Si-N layer (with reactive $\mathrm{N}_{2}$ ) is shown in Fig. 3a. Annealed at $500^{\circ} \mathrm{C}$, this sample was then electrically switched 1,000 times as shown in Fig. 3b.
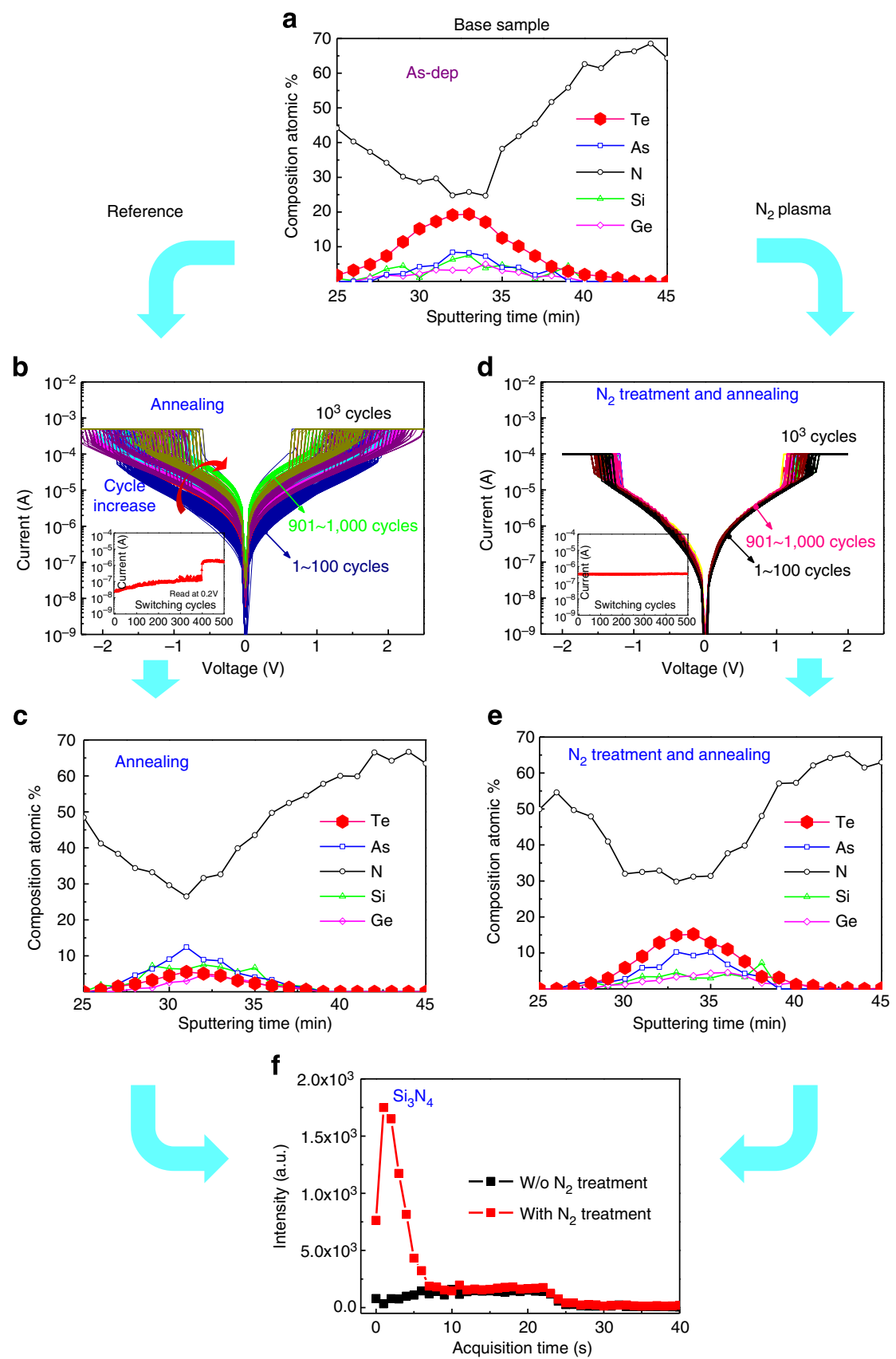

Figure 3 | XPS depth profiling. (a) Profiled as-deposited As-Ge-Te-Si-N device (with reactive $\left.\mathrm{N}_{2}\right)$. (b) Electrical cycling for 1,000 cycles $\left((0.5 \text { um })^{2}\right.$ cell size) and (c) XPS depth profile of the annealed and cycling of as-deposited As-Ge-Te-Si-N device. (d) Electrical cycling for 1,000 cycles $\left((0.5 \text { um })^{2}\right.$ cell size) and (e) XPS depth profile of the annealed $\mathrm{N}_{2}$ plasma-treated As-Ge-Te-Si-N device. (f) SIMS profile showing formation of $\mathrm{Si}_{3} \mathrm{~N}_{4}$ in the $\mathrm{N}_{2}$ plasma-treated device. Insets in $\mathbf{b}$ and $\mathbf{d}$ show the change in off-state current by switching cycle. 
a

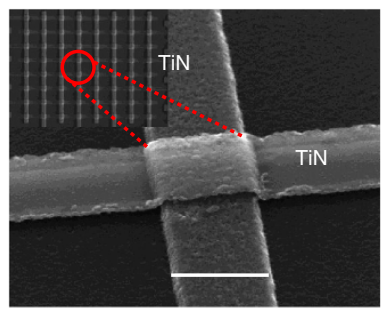

b

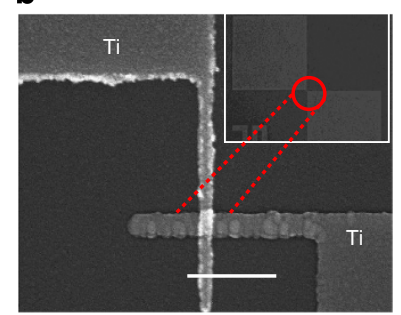

C

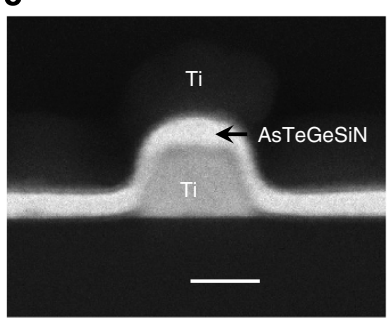

d

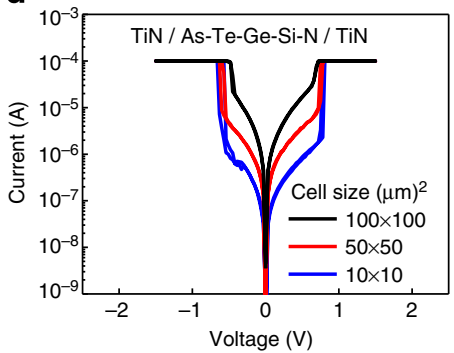

e

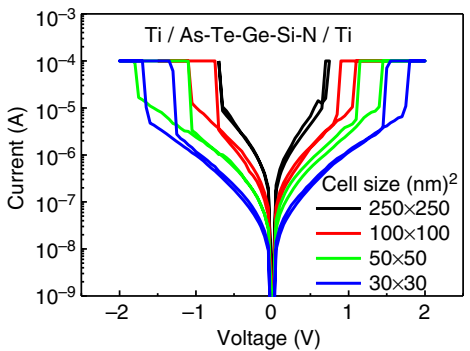

Figure 4 | Device scalability demonstrated in scanning electron microscopy and scanning transmission electron microscopy images. (a) Scanning electron microscopy (SEM) image of $(500 \mathrm{~nm})^{2}$ cell size with the inset showing an $8 \times 8$ array of $500 \mathrm{~nm}$ switch devices. Scale bar, $500 \mathrm{~nm}$. (b) SEM image of a single $(30 \mathrm{~nm})^{2}$ cell-size device. The inset shows a shrunk image with top and bottom electrodes. Scale bar, $100 \mathrm{~nm}$. (c) Cross-sectional scanning transmission electron microscopy of $(30 \mathrm{~nm})^{2}$ cell size. Scale bar, $30 \mathrm{~nm}$. (d) Switching behaviour of $(100 \mathrm{um})^{2},(50 \mathrm{um})^{2}$ and $(10 \mathrm{um})^{2}$ cell-size devices with TiN electrodes. (e) Switching behaviour of $(250 \mathrm{~nm})^{2},(100 \mathrm{~nm})^{2},(50 \mathrm{~nm})^{2}$ and $(30 \mathrm{~nm})^{2}$ cell-size devices with Ti electrodes.

The degradation with continuous switching was apparent as the off-state resistance was reduced by almost two orders of magnitude. The switching voltage also decreased on average while additionally having a wide distribution even between consecutive cycles. After cycling, we performed another XPS depth profile and were able to observe a significant change to the composition. Especially striking was the immense diffusion of the Te layer as shown in Fig. 3c from XPS results. Previous models of TS in As-Te-Ge-Si-N have proposed that Te acts as the key material for switching because the trap sites in the deep level were converted to the shallow level in the presence of high fields ${ }^{21,25-29}$. The exact effects of Te diffusion have not been considered at this time; however, it probably seems to be related to both the wide distribution and degraded device resistance properties. Before deposition of the top TiN electrode, another sample (Fig. 3d) was prepared with our $\mathrm{N}_{2}$ plasma-hardening treatment. Highly energized $\mathrm{N}_{2}$ atoms have been previously shown to increase the degree of crosslinking in $\mathrm{Si}_{3} \mathrm{~N}_{4}$ glasses ${ }^{30}$, which would retard diffusion across the region due to reduction of defect sites ${ }^{23}$. After annealing at $500^{\circ} \mathrm{C}$, we run the $\mathrm{N}_{2}$ plasma-treated device for 1,000 cycles as shown in Fig. $3 \mathrm{~d}$. The improvement in switching distribution was clear and there was almost no discernible degradation of the off-state resistance. The depth profile in XPS of the $\mathrm{N}_{2}$ plasma-treated sample in Fig. 3e displays a clear difference between the untreated and the treated samples after cycling. In the $\mathrm{N}_{2}$-treated sample, the Te region is only slightly reduced in comparison to the pristine sample of Fig. 3a. The depth profile in XPS also showed the higher $\mathrm{N}_{2}$ concentrations near the interface between the electrode and switching layer ( $\sim 30 \mathrm{~min}$ of sputtering time as shown in Fig. 3e) for the plasma-treated sample. However, it should be noted that an exact analysis of $\mathrm{N}_{2}$ content is difficult due to the existence of the TiN top electrode. We used the trap-limited conduction model ${ }^{21}$ to relate the offstate current with the total trap density and the average trap distance (Supplementary Fig. S6) within the As-Te-Ge-Si-Ndeposited films and indeed the model indicated that trap density decreased from $1.5 \times 10^{18}$ down to $4 \times 10^{17} \mathrm{~cm}^{-3}$ after plasma treatment and annealing, as shown in Supplementary Fig. S6.
Further investigation with secondary ion mass spectroscopy as shown in Fig. $3 \mathrm{f}$ reveals that $\mathrm{N}_{2}$ plasma treatment creates a thin $\mathrm{Si}_{3} \mathrm{~N}_{4}$ layer on the surface of As-Te-Ge-Si -N switch material. In addition, high-temperature XRD measurements for $\mathrm{N}_{2}$ plasma treatment show that the amorphous phase are well maintained at over $600^{\circ} \mathrm{C}$ (Supplementary Fig. S7). The contrast between the as-deposited and plasma-treated samples from the secondary ion mass spectroscopy data concludes as follows; the formation of a $\mathrm{Si}_{3} \mathrm{~N}_{4}$ barrier layer at the surface and $\mathrm{SiN}$ bonding by plasma treatment retard Te diffusion, which in turn leads to improved cycle-to-cycle disturbance. Aforementioned current density across the switch device must be sufficient for switching the $\mathrm{TaO}_{\mathrm{y}} / \mathrm{Ta}_{2} \mathrm{O}_{5-\mathrm{x}}$ memory cell into the HRS and the LRS. We found low-resistance state current to be sufficient by drastically shrinking switch devices from $(100 \mu \mathrm{m})^{2}$ all the way down to $(30 \mathrm{~nm})^{2}$ cell size to determine the scaling behaviour of the As-Ge-Te-Si-N switch devices. A 500-nm device array fabricated by photolithography is shown in Fig. $4 \mathrm{a}$ and a single $(30 \mathrm{~nm})^{2}$ cell-size device fabricated by electron beam lithography is shown in Fig. 4b. Figure $4 \mathrm{c}$ shows cross-sectional scanning transmission electron microscopy of $(30 \mathrm{~nm})^{2}$ cell size with Ti electrodes and As-Te-Ge-Si-N switch material. Measurements in Fig. $4 \mathrm{~d}$ were done on cell-size devices of $(100 \mu \mathrm{m})^{2},(50 \mu \mathrm{m})^{2}$ and $(10 \mu \mathrm{m})^{2}$, as described in Fig. 4a. Meanwhile, cell-size devices of $(250 \mathrm{~nm})^{2}$, $(100 \mathrm{~nm})^{2},(50 \mathrm{~nm})^{2}$ and $(30 \mathrm{~nm})^{2}$ in Fig. 4 e were measured in a device structure as shown in Fig. $4 \mathrm{~b}$. Ti electrodes were much less optimized for our devices than the TiN electrodes we were able to use for devices fabricated using photolithography. In fact, the leakage current for test devices fabricated at $500 \times 500 \mathrm{~nm}^{2}$ cell size showed that $\mathrm{Ti}$ electrodes increased the leakage current by almost two orders of magnitude for switches in the off-state (Supplementary Fig. S8).

\section{Discussion}

The $I-V$ behaviour across seven orders of devices size did not change significantly and the TS was observed across all devices. Interestingly, the on-current did not scale with device size up to 
the given $100 \mu \mathrm{A}$ compliances due to the filamentary nature of on-state conduction, indicating that sufficient current can be provided for electrical switching by TS switch devices. Supplementary Fig. S9 shows that even for small sizes the on-current remains the same as for larger ones. For the $30 \times 30 \mathrm{~nm}^{2}$ cell, the current density of $1.1 \times 10^{7} \mathrm{~A} \mathrm{~cm}^{-2}$ was achieved, which approaches on-current density values of singlecrystalline $\mathrm{Si}$ vertical diode at $90 \mathrm{~nm}$ technology node ${ }^{31}$. The mechanism behind filamentary TS is related to local current path formation at the threshold voltage by the electro-thermal model, which has been previously described by Kostylev ${ }^{32,33}$. Due to the highly conductive filamentary paths nature of the TS device, which is expected to be of the order of $3 \mathrm{~nm}$ diameter ${ }^{32,33}$, even devices down to $30 \mathrm{~nm}$ can provide $100 \mathrm{uA}$ current in the LRS.

Meanwhile, we measured the switching speeds for $30 \mathrm{~nm}$-sized cells with external load resistances of 10 and $50 \Omega$ for impedance matching. The input pulse and response pulse are shown in Fig. 5a. The input pulse used was $3.4 \mathrm{~V}$ with a pulse width of $100 \mathrm{~ms}$ and a rising/falling time of $1 \mathrm{~ms}$. Our best results for device switching speeds showed extremely fast switching speeds for both transitions into on- and off-states at $4 \mathrm{~ns}$ and under $2 \mathrm{~ns}$, as shown in Fig. 5b,c, respectively. It should be noted that improved manufacturing methods will be required before a largescale array could attain $4 \mathrm{~ns}$ switching speeds. The measured switching speeds were similar to those proposed by the electrothermal model ${ }^{33}$ probably indicating that plasma treatment of our samples did not affect the primary switching mechanism. Further direct current (DC) measurements showing on/off ratio 1.5 orders of magnitude and several cycles of switching for $(30 \mathrm{~nm})^{2}$ and $(70 \mathrm{~nm})^{2}$ cell sizes are presented in the Supplementary Fig. S10.

Endurance of our switch devices for $(30 \mathrm{um})^{2}$ and $(500 \mathrm{~nm})^{2}$ cells did not show any degradation up to $10^{8}$ switching cycles as shown in Fig. 6. For 30 um cells, we used on-current values limited by compliance current, whereas $500 \mathrm{~nm}$ cells were limited using an external $4 \mathrm{k} \Omega$ load resistance, as shown in Fig. 6a. The effect of load resistance for measurement of on-current is described in Supplementary Fig. S11. Figure 6a demonstrates that the DC measurement behaviour of both cells and the pulse measurement parameters (voltages and widths) are shown at the appropriate points. A $0.3-\mathrm{V}, 500-n$ s pulse was used to read off-state in both cell sizes, whereas a $2-\mathrm{V}, 500-\mathrm{ns}$ pulse was used to measure on-state in the $(30 \mathrm{um})^{2}$ cell, and a $3-\mathrm{V}, 500-\mathrm{ns}$ pulse was used to measure the on-state in the $(500 \mathrm{~nm})^{2}$ cell, respectively. We cycled our devices for up to $10^{8}$ cycles as observed in Fig. 6 b. The on/off ratio was $\sim 3$ orders and $\sim 5$ orders for $(30 \mathrm{um})^{2}$ and $(500 \mathrm{~nm})^{2}$ cells, respectively. As expected, the increased on-current density for smaller cell sizes leads to higher on/off ratios for the smaller cell sizes. Finally, we confirmed switching behaviour of cycling endurance down to $50 \mathrm{~ns}$ (Supplementary Fig. S12), which is appropriate for oxide memory materials for high-speed operation $^{3}$.

In summary, we demonstrated a switching device based on As-Ge-Te-Si material, which is significantly improved by two $\mathrm{N}_{2}$ processes: reactive $\mathrm{N}_{2}$ during deposition and $\mathrm{N}_{2}$ plasma hardening. The introduction of $\mathrm{N}_{2}$ in the above two-step processing enables a stackable and thermally stable device structure, allowing integration of switch and memory devices. Furthermore, the thin $\mathrm{Si}_{3} \mathrm{~N}_{4}$ glass layer formed by the plasma process retards $\mathrm{Te}$ diffusion during cycling leading to highly improved electrical performance. The thermal budget of $\mathrm{N}_{2}$ plasma-treated As-GeTe-Si-N in postprocessing is sufficient for stacked structures, overcoming a previous limitation of chalcogenide switching materials. In addition, electrical performance was measured down to $30 \mathrm{~nm}$ scale with extremely fast switching speed of $\sim 2 \mathrm{~ns}$ and
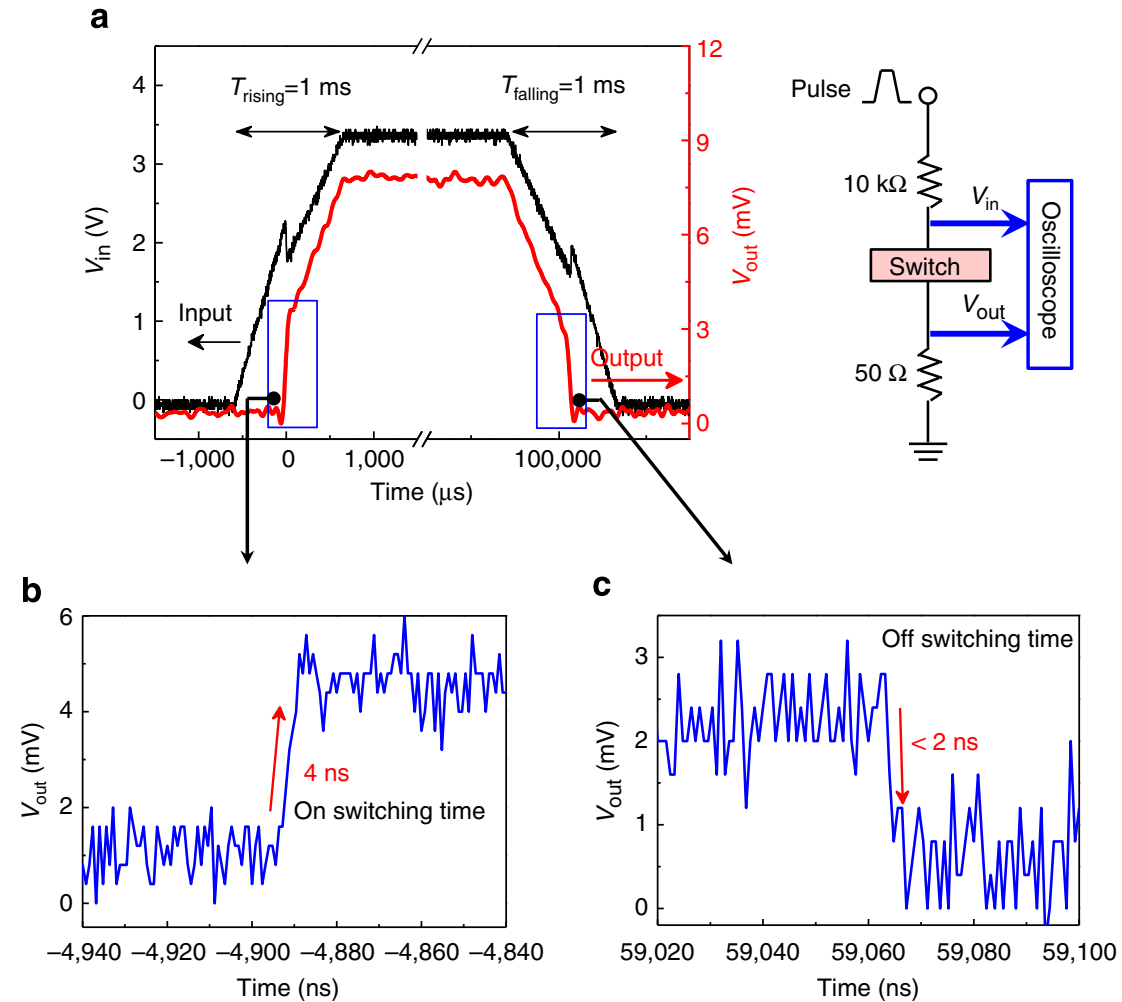

Figure 5 | Measured switching speed of As-Te-Ge-Si-N 30 nm-sized switch device. (a) Real-time oscilloscope response measuring switching speed with external circuit schematics used detailed on right. (b) Zoomed-in response at the instant from off-state to on-state. Switching time was 4 ns. (c) Zoomed-in response at the instant from on-state to off-state. Switching time was under $2 \mathrm{~ns}$. 
a

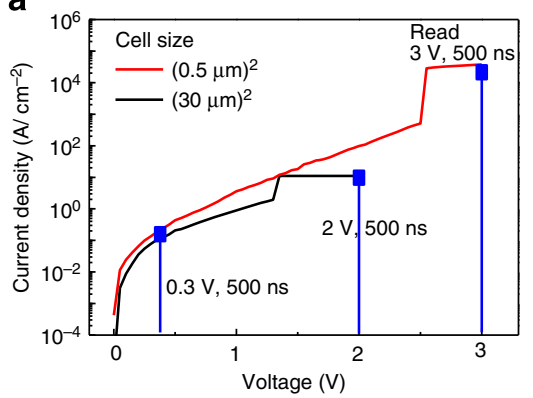

b

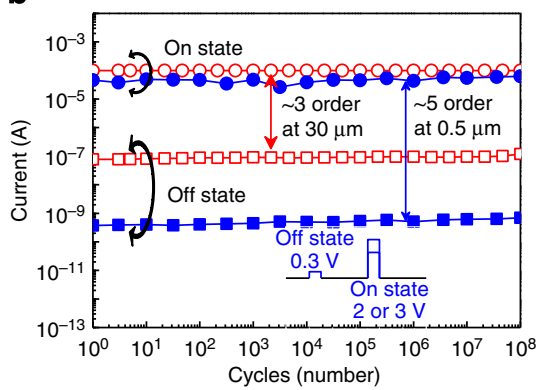

Figure 6 | Cycling endurance in As-Ge-Te-Si-N switch devices. (a) DC I-V measurement of the (30 um) $)^{2}$ cell-size device using compliance current and $(500 \mathrm{~nm})^{2}$ cell-size device using external $4 \mathrm{k} \Omega$ load resistance. Pulse is measured with reading voltages for on/off-states indicated by drop lines. (b) Cycling endurance up to $10^{8}$ shown for $(30 \mathrm{um})^{2}$ and $(500 \mathrm{~nm})^{2}$ cell-size devices. The inset shows a diagram of voltage pulses for testing this scheme.

proved to be satisfactory for nanoscale memory applications in high-density integration.

\section{Methods}

Electrical pulse measurements. The DC electrical measurements of test cells were performed by using an Agilent 4156C Semiconductor Parameter Analyzer. Pulse measurements for cycling endurance were conducted by an Agilent $81110 \mathrm{~A}$ pulse generator and Tektronix oscilloscope $(6 \mathrm{GHz})$. For cycling endurance measurements, an external load resistance was serially connected between the device and the analyser. Uniformity of samples across a $150-\mathrm{mm}$ wafer was measured using DC measurements to cycle respective devices 100 times each (Supplementary Fig. 13).

Preparation of memory and switch devices. We fabricated both $\mathrm{W} / \mathrm{AlO}_{\mathrm{x}} / \mathrm{TaO}_{\mathrm{y}} /$ $\mathrm{Ta}_{2} \mathrm{O}_{5-\mathrm{x}} / \mathrm{Pt}$ resistor structures as memory devices and TiN/As-Te-Ge-Si-N/TiN or Ti structure as bidirectional switch devices. All films were deposited by using reactive DC magnetron sputtering based on the respective metal target. For the fabrication of memory devices, $\mathrm{W}$ bottom electrode was deposited. Next, the $\mathrm{TaO}_{\mathrm{y}}$ layer is deposited and the insulating $\mathrm{Ta}_{2} \mathrm{O}_{5-\mathrm{x}}$ layer was subsequently formed by placing our samples in an $\mathrm{O}_{2}$ plasma oxidation chamber used for atomic layer deposition and capped by top Pt electrode. After that, the TiN (Ti for cell sizes below $250 \mathrm{~nm}$ ) switch contact was formed by reactive sputter of a Ti target in a mixture of $\mathrm{N}_{2}$ and Ar. Then, a 40-nm switch layer of As-Ge-Te-Si-N was deposited using reactive sputter of an As-Te-Ge-Si target again in a mixture of $\mathrm{N}_{2}$ and Ar gas, and a $\mathrm{N}_{2}$ plasma treatment was formed and capped with the top TiN or Ti electrode (for cell sizes below $250 \mathrm{~nm}$ ). E-beam lithography was used to define crossbar cell structures of switch devices from 250 to $30 \mathrm{~nm}$ sizes. First, a two-layered electroresist fabrication process was used to create Ti bottom electrode lines ( $40 \mathrm{~nm}$ thick) on the 500 -nm thick $\mathrm{SiO} 2$ substrates. The two electroresist layers were composed of ZEP-520A7 from ZEONREX Electronic Chemicals on top of Lift-offResist 1A (LOR1A) from Microchem Corp. This method was used to define a more precise cell structure to achieve accurate measurements and higher device yields. Top Ti deposition was performed using E-beam evaporation after pattering the bottom lines by E-beam lithography and then using a lift-off method. Device sizes from $(0.5 \mathrm{um})^{2}$ and above were fabricated similar to E-beam samples; however, conventional projection photolithography was used. Annealing conditions for the experiments presented in Fig. 2 were performed within the sputtering chamber. First the chamber was pumped down to a base pressure of $10^{-7}$ Torr and the substrate holder temperature was increased to $500^{\circ} \mathrm{C}$. The samples were annealed from 15 to $30 \mathrm{~min}$ and then removed. In addition, annealing in $\mathrm{N}_{2}$ ambient at 30 mTorr under the same temperature was performed, however, device perfor mance for both reactive $\mathrm{N}_{2}$ devices and pristine devices did not show any difference from those of vacuum-annealed samples.

Trap-limited conduction model. We performed the modelling for off-state conduction based on the trap-limited conduction (TLC) mechanism ${ }^{21}$ for an As-Te-Ge-Si-N chalcogenide glass. From the TLC model (ref. 21), the current (I) can be described as

$$
I=2 q A N_{T} \frac{\Delta z}{\tau_{0}} e^{-\left(E_{C}-E_{F}\right) / k T} \sinh \left(\frac{q V_{A}}{k T} \frac{\Delta z}{2 u_{a}}\right),
$$

where $q$ is the elementary charge, $A$ is the area of the contact, $N_{T}$ is the integral of the trap distribution in the gap above the Fermi level, $\Delta z$ is the average distance between two traps, $\tau_{0}$ is the characteristic attempt-to-escape time for the trapped electron $\left(\sim 10^{-13} s\right), E_{\mathrm{C}}$ is the energy at the conduction band edge, $E_{\mathrm{F}}$ is the Fermi level, $V_{A}$ is the applied voltage and $u_{\mathrm{a}}$ is the amorphous chalcogenide thickness $(\sim 50 \mathrm{~nm})$. As the real trap distribution in the chalcogenide material is generally not known, we will neglect the inaccuracies of equation 1 for $E_{\mathrm{C}}$ and $E_{\mathrm{F}}$ and will treat $N_{T}$ as an effective trap concentration.

By using the TLC model ${ }^{21}$, we extracted the total trap density $\left(N_{T}\right)$ and inter-trap distance $(\Delta z)$ at experimental switching data $\left(10^{3}\right.$ cycles $)$ of various process conditions as shown in Supplementary Fig. S6. Compared with the as-deposited sample, the change in trap density and distance of $\mathrm{N}_{2}$-treated and annealed one was suppressed, which means trap density and distance are strongly related with Te loss.

\section{References}

1. International Technology Roadmap for Semiconductors: ITRS 2011 EditionEmerging Research Devices ITRS (2011).

2. Cario, L., Vaju, C., Corraze, B., Guiot, V. \& Janod, E. Electric-field-induced resistive switching in a family of Mott insulators: towards a new class of RRAM memories. Adv. Mater. 22, 5193-5197 (2010).

3. Lee, M.-J. et al. A fast, high-endurance and scalable non-volatile memory device made from asymmetric $\mathrm{Ta}_{2} \mathrm{O}_{5-\mathrm{x}} / \mathrm{TaO}_{2-\mathrm{x}}$ bilayer structures. Nat. Mater. 10, 625-630 (2011).

4. Borghetti, J. et al. 'Memristive' switches enable 'stateful' logic operations via material implication. Nature 464, 873-876 (2010).

5. Strukov, D. B., Snider, G. S., Stewart, D. R. \& Williams, R. S. The missing memristor found. Nature 453, 80-83 (2008).

6. Yang, J. J., Borghetti, J., Murphy, D., Stewart, D. R. \& Williams, R. S. A family of electronically reconfigurable nanodevices. Adv. Mater. 21, 3754-3758 (2009).

7. Waser, R., Dittmann, R., Staikov, G. \& Szot, K. Redox-based resistive switching memories-nanoionic mechanisms, prospects, and challenges. Adv. Mater. 21, 2632-2663 (2009).

8. Lee, M.-J. et al. A low-temperature-grown oxide diode as a new switch element for high-density, nonvolatile memories. Adv. Mater. 19, 73-76 (2007).

9. Linn, E., Rosezin, R., Kugeler, C. \& Waser, R. Complementary resistive switches for passive nanocrossbar memories. Nat. Mater. 9, 403-406 (2010).

10. Waser, R. \& Aono, M. Nanoionics-based resistive switching memories. Nat Mater. 6, 833-840 (2007).

11. Seo, S. et al. Reproducible resistance switching in polycrystalline $\mathrm{NiO}$ films. Appl. Phys. Lett. 85, 5655-5657 (2004).

12. Lee, M.-J. et al. Electrical manipulation of nanofilaments in transition-metal oxides for resistance-based memory. Nano. Lett. 9, 1476-1481 (2009).

13. Liu, S. Q., Wu, N. J. \& Ignatiev, A. Electric-pulse-induced reversible resistance change effect in magnetoresistive films. Appl. Phys. Lett. 76, 2749-2751 (2000).

14. Baek, I. G. et al. Highly scalable non-volatile memory using simple binary oxide driven by asymmetric unipolar voltage pulses. IEEE Int. Electron Dev. Meet. Tech. Dig. 04, 587-590 (2004).

15. Park, W. Y. et al. A Pt/TiO $/ 2$ Ti Schottky-type selection diode for alleviating the sneak current in resistance switching memory arrays. Nanotechnology 21, 195201 (2010).

16. Lee, W. et al. Varistor-type bidirectional switch $\left(\mathrm{J}_{\mathrm{MAX}}>10^{7} \mathrm{~A} / \mathrm{cm}^{2}\right.$, swlwctivity $\sim 10^{4}$ ) for 3D bipolar resistive memory arrays. Symposium on VLSI Technology Digest of Technical Papers 2012, 37-38 (2012).

17. Burr, G. W. et al. Large-scale ( $512 \mathrm{kbit})$ integration of multilayer-ready accessdevices based on mixed-ionic-electronic-conduction (MIEC) at $100 \%$ yield. Symposium on VLSI Technology Digest of Technical Papers 2012, 41-42 (2012).

18. Son, M. et al. Excellent selector characteristics of nanoscale $\mathrm{VO}_{2}$ for highdensity bipolar ReRAM applications. IEEE Electron Dev. Lett. 32, 1579-1581 (2011).

19. Austin, I. G. \& Mott, N. F. Metallic and nonmetallic behavior in transition metal oxides. Science 168, 71-77 (1970). 
20. Lee, M.-J. et al. A simple device unit consisting of all NiO storage and switch elements for multilevel terabit nonvolatile random access memory. ACS Appl. Mater. Interfaces 3, 4475-4479 (2012).

21. Ielmini, D. \& Zhang, Y. Analytical model for subthreshold conduction and threshold switching in chalcogenide-based memory devices. J. Appl. Phys. 102, 054517 (2007)

22. Ovshinsky, S. R. Reversible electrical switching phenomena in disordered structures. Phys. Rev. Lett. 21, 1450-1455 (1968).

23. Chen, G. \& Chen, J. Role of nitrogen in the crystallization of silicon nitridedoped chalcogenide glasses. J. Am. Ceram. Soc. 82, 2934-2936 (1999).

24. Lee, J. H. et al. Threshold switching in Si-As-Te thin film for the selector device of crossbar resistive memory. Appl. Phys. Lett. 100, 123505 (2012).

25. Karpov, V. G., Kryukov, Y. A., Savransky, S. D. \& Karpov, I. V. Nucleation switching in phase change memory. Appl. Phys. Lett. 90, 123504 (2007).

26. Karpov, V. G., Kryukov, Y. A., Karpov, I. V. \& Mitra, M. Field-induced nucleation in phase change memory. Phys. Rev. B 78, 052201 (2008).

27. Redaelli, A., Pirovano, A., Benvenuti, A. \& Lacaita, A. L. Threshold switching and phase transition numerical models for phase change memory simulations. J. Appl. Phys. 103, 111101 (2008).

28. Kastner, M., Adler, D. \& Fritzsche, H. Valence-alternation model for localized gap states in lone-pair semiconductors. Phys. Rev. Lett. 37, 1504-1507 (1976).

29. Adler, D., Henisch, H. K. \& Mott, N. The mechanism of threshold switching in amorphous alloys. Rev. Mod. Phys. 50, 209-220 (1978).

30. Chen, G., Chen, J. J. \& Chen, W. Raman spectra of Ge-Si-As-Se-Te-N. Phys. Chem. Glasses 38, 335-337 (1997).

31. Oh, J. H. et al. Full integration of highly manufacturable $512 \mathrm{Mb}$ PRAM based on $90 \mathrm{~nm}$ technology. IEEE Int. Electron Dev. Meet. Tech. Dig. 06, 515-518 (2006).
32. Kostylev, S. A. Threshold and filament current densities in chalcogenide-based switches and phase-change-memory devices. IEEE Electron Dev. Lett. 30, 814-816 (2009).

33. Kostylev, S. A. Dimensionality effects in chalcogenide-based devices. Physica E 51, 120-127 (2013).

\section{Acknowledgements}

We are grateful to SAIT colleagues Y.K. Cha, K.J. Park and C.Y. Moon for their assistance with experiments and J.-B. Park for TEM analysis assistance, Kyunghee University and K.R. Park for photograph imaging assistance.

\section{Author contributions}

M.-J.L., D.S. and S.-H.C designed this work and prepared the manuscript. The experiment and electrical measurements were carried out by M.-J.L. and D.S. All authors discussed the results and implications and commented on the manuscript at all stages.

\section{Additional information}

Supplementary Information accompanies this paper at http://www.nature.com/ naturecommunications

Competing financial interests: The authors declare no competing financial interests.

Reprints and permission information is available online at http://npg.nature.com/ reprintsandpermissions/

How to cite this article: Lee, M.-J. et al. A plasma-treated chalcogenide switch device for stackable scalable 3D nanoscale memory. Nat. Commun. 4:2629 doi: $10.1038 /$ ncomms3629 (2013). 